\title{
Evaluation of 3 (4, 5 dimethyl-2-thiazolyl)-2, 5 diphenyl tetrazolium bromide method for rapid detection of vancomycin-resistant enterococci
}

\author{
Nucharat Panthasri $^{\mathrm{a}}$, Prajuab Chaimanee ${ }^{\mathrm{b}}$, Suthida Kenprom ${ }^{\mathrm{b}}$, Patcharaporn Tippayawat ${ }^{\mathrm{c}}$, \\ Nicha Charoensric ${ }^{\mathrm{c}}$, Aroonwadee Chanawong ${ }^{\mathrm{c}}$, Ratree Tavichakorntrakool ${ }^{\mathrm{c}}$, Aroonlug Lulitanond ${ }^{\mathrm{c}, *}$ \\ a The 8th Regional Medical Science Center, Udon Thani 41330 Thailand \\ b Clinical Microbiology Unit, Srinagarind Hospital, Khon Kaen University, Khon Kaen 40002 Thailand \\ c Centre for Research and Development of Medical Diagnostic Laboratories (CMDL), Faculty of Associated \\ Medical Sciences, Khon Kaen University, Khon Kaen 40002 Thailand
}

*Corresponding author, e-mail: arolul@kku.ac.th

Received 15 Jul 2020

Accepted 14 Oct 2020

\begin{abstract}
Vancomycin-resistant enterococci (VRE) have become a global problem of hospital-associated infections. A rapid VRE detection and early isolation of the positive cases are essential for prevention and control of their transmission in hospitals. The aim of this study was to develop and evaluate a simple and rapid method for detection of VRE by using 3 (4, 5 dimethyl-2-thiazolyl)-2, 5 diphenyl tetrazolium bromide (MTT) colorimetric method compared with standard broth microdilution (BMD) and Epsilometer test (Etest). A total of 85 Enterococcus isolates (75 vancomycinnon-susceptible, 2 vancomycin-intermediate and 8 vancomycin-susceptible) were studied. The minimum inhibitory concentrations (MICs) of vancomycin for all isolates were tested by BMD, MTT and Etest methods. The MTT method gave results within $9 \mathrm{~h}$ with good category agreement, essential agreement, very major, major, and minor errors (100, 92.9, 0, 0 and 7.1\%, respectively), which were comparable to those of the Etest $(97.6,95.3,2.4,0$ and 4.7\%, respectively). The Cohen's kappa coefficient $(\kappa)$ of the MTT method compared with the BMD showed perfect agreement (1.000) $(p<0.001)$. The MTT method is simple and rapid for early VRE detection to support prevention and control the spread of the enterococci in hospitals.
\end{abstract}

KEYWORDS: vancomycin resistance, enterococci, Etest, broth microdilution, MTT

\section{INTRODUCTION}

Enterococci are common bacteria found in gastrointestinal tract of humans and animals, soil, foods, and water. They cause various infections in humans including endocarditis, intra-abdominal infections, pelvic infections, urinary tract infections, skin infections, and central nervous system infections [1]. Most enterococcal infections in humans were caused by Enterococcus faecalis with the rate accounted around 80\%, while Enterococcus faecium caused about 20\% of the infections [2]. Enterococci are intrinsically resistant to several common antimicrobials and have high potential to acquire antimicrobial resistance factors, which cause serious problems in patient therapy $[1,3]$. In the recent decades, the emergence of vancomycin-resistant enterococci (VRE) has become a global public health concern $[4,5]$.

There are several acquired determinants, which are responsible for glycopeptide resistance in enterococci. These include vanA, vanB, vanD, vanE, van $G$, vanL, vanM, and vanN. The vanA and vanB are two vancomycin resistance gene clusters of clinical relevance, which are located on the transposable elements, Tn1546 and Tn1549 [6,7], being transferred between strains by plasmids and conjugative transposons. The vanA was responsible for most of VRE in human cases around the world [8]. It was predominantly carried by $E$. faecium, followed by E. faecalis, whereas for the non-transferable, vanC was a unique intrinsic resistance in E. gallinarum and E. casseliflavus [4]. The ability of transferable vanA and vanB led to the emerging of VRE [9]. In addition, bacteria carrying vanA usually have high minimum inhibitory concentrations (MICs) of vancomycin (>64 $\mu \mathrm{g} / \mathrm{ml})$, leading to a high-level resistance in most clinical VRE. In contrast, the intrinsic vancomycin resistance E. casseliflavus and E. gallinarum, carrying vanC, have low MICs of 
vancomycin $(\leqslant 32 \mu \mathrm{g} / \mathrm{ml})$ [4].

The vancomycin susceptibility test for Enterococcus spp. by a disk diffusion method was performed by incubation for $24 \mathrm{~h}$. Subsequently, the inhibition zones should be observed carefully with transmitted light for small colonies or haze inside the zone. Any growth found in the inhibition zone may indicate the resistance [10]. The vancomycin-intermediate enterococci and VRE should be then confirmed by using vancomycin agar screen plate method or broth microdilution method (BMD) for their MIC determination. Intermediate resistance to vancomycin is usually found among E. gallinarum and E. casselifavus [11]. When VRE was detected, it should be reported immediately for prompt infection control in the hospital [12].

Several studies have reported on VRE detection. The vanA-carrying VRE were detected by both conventional disk diffusion or agar dilution, and automatic system such as Vitek GPS-101 and MicroScan system, whereas some vanB-carrying VRE were not detected by the automatic system [13]. The agar screen test was the most reliable and an easy method for routine screening; but it took up to $24 \mathrm{~h}$. The modern methods, such as Matrix-Assisted Laser Desorption Ionization-Time of Flight Mass Spectrometry (MALDI-TOF MS) and real time PCR, gave results more rapidly; but they needed specific experiences and expensive equipments [14-16].

3 (4, 5 dimethyl-2-thiazolyl)-2, 5 diphenyl tetrazolium bromide (MTT) is a yellow chemical substance, which becomes blue formazan when dehydrogenated by mitochondria of living cells [17]. The MTT assay had been applied for assessing cell metabolic activity, cytotoxicity or loss of viable cells [17-19]. Vancomycin is a bactericidal agent which kills the susceptible bacterial cells. Therefore, we applied the MTT in BMD method for VRE testing to shorten the incubation time.

This study aimed to evaluate an MTT method for detection of VRE compared with standard broth microdilution (BMD) and Etest. The information would be useful for VRE detection and control in hospitals with low resources.

\section{MATERIALS AND METHODS}

A total of 85 Enterococcus isolates (77 vancomycin non-susceptible Enterococcus and 8 vancomycinsusceptible Enterococcus) collected between January and December 2017 from patients of hospitals in the upper area of northeastern Thailand were studied. They were identified by biochemical tests and kept at $-20{ }^{\circ} \mathrm{C}$ in skimmed milk plus $20 \%$ glycerol.
Reference strains of vancomycin-resistant $E$. faecalis, E. faecium, E. gallinarum, and E. casseliflavus, carrying van $A$, vanB, vanC1, and vanC2 genes, respectively, and vancomycin-susceptible $E$. faecalis ATCC 29212 were used as control strains.

\section{Ethics approval}

This study was conducted in accordance with the Declaration of Helsinki and was approved by the Ethics Committee of Khon Kaen University (project no. HE591546).

\section{MIC determination}

The MICs of vancomycin (Sigma-Aldrich, St. Louis, USA) for all isolates were determined by BMD method using vancomycin concentrations ranging from 0.125 to $1024 \mu \mathrm{g} / \mathrm{ml}$ according to the standard CLSI [10]. In addition, the Etest MICs of vancomycin for all isolates were performed according to the manufacturer description (AB Biodisk, Solna, Sweden). The results were interpreted according to the CLSI [10].

\section{MTT method}

The MICs of vancomycin were determined by rapid MTT method in a 96 well microplate. The vancomycin concentrations ranging from 0.125 to $1024 \mu \mathrm{g} / \mathrm{ml}$ were mixed with $10^{5} \mathrm{CFU} / \mathrm{ml}$ of fresh culture of each isolate and incubated for $8 \mathrm{~h}$ at $37^{\circ} \mathrm{C}$. After the incubation, $20 \mu \mathrm{l}$ of MTT solution $(5 \mathrm{mg} / \mathrm{ml}$ in phosphate buffer) (Sigma-Aldrich, St. Louis, USA) were added to each well and reincubated for $1 \mathrm{~h}$. The formation of blue color in the solution indicated the presence of viable bacterial cells [18]. The MICs were determined by visual minimum drug concentration that still had yellow color. The MTT test was carried out in triplicate for each isolate.

The time-kill value of Enterococcus for vancomycin from a previous study was around $8 \mathrm{~h}$ [20], thus in this study, the bacteria were exposed to vancomycin for $8 \mathrm{~h}$ before detection with the MTT solution.

\section{Detection of species specific and van genes}

The total bacterial DNA was prepared by a rapid alkaline lysis method as described previously [21]. The Enterococcus species specific for E. faecium, E. faecalis, and the vanA, vanB and vanC1 genes (for E. gallinarum) were amplified by multiplex polymerase chain reaction (PCR) using oligodeoxynucleotide primers according to a previous report [22]. 


\section{Data analysis}

The results were evaluated in terms of the following parameters: categorical agreement (CA, the total number of isolates tested that yielded the same categorical interpretation as the reference method) and essential agreement (EA, the obtaining MIC value was within $1 \log _{2}$ dilution of the reference value). The CA is subdivided into 3 types of error: very major errors, major errors, and minor errors. A very major errors was defined as an isolate that was resistant by the reference BMD method, but susceptible with the test method (false susceptibility). A major error was defined as an isolate that was susceptible by the reference BMD method, but resistant with the test method (false resistance). A minor error was defined as a discrepancy between the results of the reference BMD and the test method corresponding to one interpretation category. The acceptable rate for $\mathrm{CA}$ and $\mathrm{EA}$ is $\geqslant 90 \%$. Acceptable minor error rates are $\leqslant 10 \%$, while acceptable major error and very major errors rates are $<3 \%$ of the susceptible and resistant isolates tested, respectively [23].

Statistical analysis was performed using SPSS version 13 (Statistical Package for the Social Sciences, Chicago, IL, USA). Cohen's kappa coefficient test was used to compare the MIC results between the standard BMD method and the MTT method. Kappa value was calculated to analyse the level of agreement between the standard BMD method and MTT method. A probability of $<0.05$ was considered significant.

\section{RESULTS}

\section{Species identification}

A total of 85 catalase-negative, gram-positive cocci were subjected for biochemical tests and confirmed by PCR technique. The 85 Enterococcus isolates were identified as 80 E. faecium (94.1\%), 3 E. faecalis (3.5\%), 1 E. gallinarum (1.2\%) and 1 non-specified Enterococcus species (1.2\%).

\section{Determination of vancomycin MIC and detection of vancomycin resistance genes}

The MICs of vancomycin for the 85 Enterococcus isolates determined by standard BMD method showed 75 vancomycin-resistant enterococci (VRE) (88.2\%), 2 vancomycin-intermediate $(2.4 \%)$, and 8 vancomycin-susceptible enterococci $(9.4 \%)$. All of the 75 VRE isolates were E. faecium carrying vanA, which had high vancomycin MICs of 128 to $>1024 \mu \mathrm{g} / \mathrm{ml}$. Eight Enterococcus isolates had low vancomycin MICs $(1-4 \mu \mathrm{g} / \mathrm{ml})$ and no vanA gene. One isolate of E. gallinarum had low vancomycin MIC of $16 \mu \mathrm{g} / \mathrm{ml}$. Most of E. faecalis were vancomycin-susceptible.

\section{Comparison of MICs from MTT method and Etest with BMD method}

The results obtained from the MTT method and Etest versus those of the standard BMD were shown in Table 1. The CA, EA, VME, ME, and $\mathrm{mE}$ of the MTT method were within the acceptance criteria (100, 92.9, 0, 0, and $7.1 \%$, respectively), and were comparable to those of the Etest $(97.6,95.3,2.4$, 0 and $4.7 \%$, respectively). The error rates of both methods were shown in Table 2. The Cohen's kappa coefficient $(\kappa)$ of MTT method compared with BMD showed perfect agreement $(\kappa=1.000 ; p<0.001)$. The correlations between the Etest and the BMD method was almost perfect with Cohen's kappa coefficient $(\kappa)=0.88(p<0.001)$.

\section{DISCUSSION}

VRE have been reported increasingly and become a major problem in nosocomial infections [24]. It caused a significant increasing rate of bloodstream infection from 5.9 to $16.7 \%$ during a 10 year surveillance of a hospital in Germany [25]. The prevalence of VRE had also been increased in other countries. In China, a 2015 report from Beijing showed up to $14.3 \%$ increasing incidence of VREE. faecium, and $1.3 \%$ of VRE-E. faecalis [26]. In Thailand, most VRE were E. faecium carrying vanA. The annual report in Thailand between 2014-2016 showed increasing VRE infection rates of 1.7, 2.2 and $3.6 \%$, respectively. The rates of VRE-E. faecium were $4.3,4.5$ and 9.9\%, while those of VRE-E. faecalis were $0.3,1.3$ and $0.4 \%$, respectively [27]. In addition, most of the Enterococcus isolates in Thailand with high MICs of vancomycin carried vanA, similar to those in China [26]. In contrast, most VRE isolates in Australia were E. faecium, which related to $\operatorname{vanB}[21]$.

The prevention and control of VRE transmission in hospitals require an early detection of infected or colonized patients. It is crucial to have a rapid VRE detection and early isolation of the positive cases. Therefore, the rapid and accurate detection of vancomycin resistance is essential. In this study we evaluated a simple rapid method for detection of VRE compared with the standard BMD method and Etest. The MTT assay has become a gold standard for determination of cell viability and proliferation since 1983 [28]. The assay measures cell viability from reductive activity of enzymes that converses 
Table 1 Comparison of vancomycin MICs of Enterococcus spp. by BMD, MTT and Etest methods.

\begin{tabular}{lccccccccccc}
\hline \multirow{2}{*}{ Method } & \multicolumn{10}{c}{ Number of Enterococcus spp. with MIC of vancomycin $(\mu \mathrm{g} / \mathrm{ml})$} \\
\cline { 2 - 11 } & 1 & 2 & 4 & 8 & 16 & 32 & 64 & 128 & 256 & 512 & 1024 \\
\hline BMD & $1 *$ & $6 *$ & $1 *$ & 1 & 1 & 0 & 0 & 2 & 6 & 45 & 22 \\
MTT & $2 *$ & $6 *$ & $6^{*}$ & 1 & 1 & 0 & 0 & 2 & 10 & 42 & 21 \\
Etest & $8 *$ & $0^{*}$ & $2 *$ & 0 & 0 & 0 & 0 & 0 & 0 & 75 & 0 \\
\hline
\end{tabular}

* indicates the vancomycin-susceptible isolates.

Table 2 Error rates and agreements of MTT and Etest methods for detection of vancomycin-resistant Enterococci compared to BMD method.

\begin{tabular}{|c|c|c|c|c|c|}
\hline \multirow[t]{2}{*}{ Method } & \multicolumn{3}{|c|}{ Error rate $(\%)^{\mathrm{a}}$} & \multirow{2}{*}{$\begin{array}{c}\text { Category } \\
\text { agreement (\%) }\end{array}$} & \multirow{2}{*}{$\begin{array}{c}\text { Essential } \\
\text { agreement (\%) }\end{array}$} \\
\hline & Very major & major & minor & & \\
\hline MTT & $0(0)$ & $0(0)$ & $6(7.1)$ & 100 & 92.9 \\
\hline Etest & $2(2.4)$ & $0(0)$ & $4(4.7)$ & 97.6 & 95.3 \\
\hline Acceptance criteria & $<3 \%$ & $<3 \%$ & $\leqslant 10 \%$ & $\geqslant 90 \%$ & $\geqslant 90 \%$ \\
\hline
\end{tabular}

${ }^{a}$ Relative to the CLSI reference BMD method; very major error, false susceptibility; major errors, false resistance; minor errors, a discrepancy between the results of the reference method and the test method corresponding to one interpretation category.

the tetrazolium compound to water insoluble formazan crystals. The MTT has been used as an indicator to evaluate cell proliferation and microbial growth $[29,30]$. For example, an MTT assay for rapid detection of rifampicin-resistant Mycobacterium tuberculosis gave high sensitivity, specificity, positive and negative predictive values of $100 \%$, $86 \%, 100 \%, 99 \%$, respectively [31]. In the present study, the MICs of vancomycin for Enterococcus using MTT method gave congruent results to those of the reference BMD method. The CA and EA were over $90 \%$ and the VME, ME and $\mathrm{mE}$ were within the acceptance criteria. Similarly, Etest also gave good results with the CA, EA, VME, ME, and mE values within the acceptance criteria. Etest is an easy method for MIC determination without performing the serial dilution. However, it needed to incubate for $24 \mathrm{~h}$ to detect the vancomycin resistance, while the MTT method in this study needed a shorter period of incubation.

The time-kill value of Enterococcus for vancomycin from a previous study was around $8 \mathrm{~h}$ [20], thus in this study the bacteria were exposed to vancomycin for $8 \mathrm{~h}$ before detection with the MTT solution. The MICs of vancomycin for both VRE and non-VRE isolates were clearly observed by naked eye within one day. This method is simple and fast. It does not require any sophisticated equipments or additional tests to confirm the antibiotic resistance. The cost per test is 1.21 USD (for triplicate test).
The present study was a primary test; thus the MTT concentration was not optimized but followed a previous report [18]. Therefore, further study with more samples and optimization of MTT concentrations should be performed. This MTT method should be used carefully when testing with slowgrowth rate strains. The MTT reagent is easy to prepare and has a long shelf life. Stored properly at $4^{\circ} \mathrm{C}$ and protected from lights, the kit components would remain stable for 12 months. For in-house MTT solution, once prepared, the solution when stored for four weeks at $4{ }^{\circ} \mathrm{C}$ and protected from lights can be used. The DMSO-formazan solution stored in the dark would provide stable absorption readings for up to $1 \mathrm{~h}$. In this study, the optical density of bacterial growth at $520 \mathrm{~nm}$ of each well was $>0.24$; whereas those with no bacterial growth were $<0.24$, which was clearly consistent to those inspected by naked-eye (data not showed). Therefore, it is appropriate for VRE prevention and control in low-resource settings.

\section{CONCLUSION}

The vancomycin susceptibility for Enterococcus was performed by using MTT colorimetric method. The result was obtained within $9 \mathrm{~h}$ and in concordance with the standard BMD method (Cohen's kappa coefficient $(\kappa)=1.000)$. This method may be a rapid and economical test for routine service. Most VRE isolates in this area were vanA-carrying E. faecium. 
Acknowledgements: This work was supported by Khon Kaen University (grant no. 6200017). We are grateful to Prof. Keiichi Hiramatsu for providing the reference strains, and to staff of Clinical Microbiology Unit, Srinagarind Hospital and hospitals in the upper area of northeastern Thailand for collecting the clinical isolates.

\section{REFERENCES}

1. Suvarna K, Mahon CR (2019) Streptococcus, Enterococcus, and other catalase-negative, gram-positive cocci. In: Mahon CR, Lehman DC (eds) Textbook of Diagnostic Microbiology, 6th edn, Elsevier Saunders, St. Luis, USA, pp 322-343.

2. Hidron AI, Edwards JR, Patel J, Horan TC, Sievert DM, Pollock DA, Fridkin SK (2008) NHSN annual update: antimicrobial-resistant pathogens associated with healthcare-associated infections: annual summary of data reported to the National Healthcare Safety Network at the Centers for Disease Control and Prevention, 2006-2007. Infect Control Hosp Epidemiol 29, 996-1011.

3. Eliopoulos GM, Gold HS (2001) Vancomycinresistant enterococci: Mechanisms and clinical observations. Clin Infect Dis 33, 210-219.

4. Weiner LM , Webb AK, Limbago B, Margaret A, Dudeck MA (2016) Antimicrobial-resistant pathogens associated with healthcare-associated infections: summary of data reported to the National Healthcare Safety Network at the Centers for Disease Control and Prevention, 2011-2014. Infect Control Hosp Epidemiol 37, 1288-1301.

5. Humphreys H (2014) Controlling the spread of vancomycin-resistant enterococci. Is active screening worthwhile? J Hosp Infect 88, 191-198.

6. Arthur M, Molinas C, Depardieu F, Courvalin P (1993) Characterization of Tn1546, a Tn3-related transposon conferring glycopeptide resistance by synthesis of depsipeptide peptidoglycan precursors in Enterococcus faecium BM4147. J Bacteriol 175, 117-127.

7. Wardal E, Markowska K, Żabicka D, Wróblewska M, Giemza M, Mik E, Połowniak-Pracka H, Woźniak A, et al (2014) Molecular analysis of vanA outbreak of Enterococcus faecium in two Warsaw hospitals: the importance of mobile genetic elements. Biomed Res Int 2014, ID 575367.

8. Miller WR, Munita JM, Arias CA (2014) Mechanisms of antibiotic resistance in enterococci. Expert Rev Anti Infect Ther 12, 1221-1236.

9. Werner G, Coque TM, Hammerum AM, Hope R, Hryniewicz W, Johnson A, Klare I, Kristinsson KG, et al (2008) Emergence and spread of vancomycin resistance among enterococci in Europe. Euro Surveill 13, ID 19046.

10. CLSI (2017) Performance Standard for Antimicrobial Susceptibility Testing, 27th edn, M100, CLSI, PA, USA.
11. Praharaj I, Sujatha S, Parija SC (2013) Phenotypic \& genotypic characterization of vancomycin resistant Enterococcus isolates from clinical specimens. Indian $J$ Med Res 138, 549-556.

12. HICPAC (1995) Recommendations for preventing the spread of vancomycin resistance: recommendations of the Hospital Infection Control Practices Advisory Committee (HICPAC). Am J Infect Control 23, 87-94.

13. Endtz HP, Braak Van DN, Belkum A Van, Goessens WH, Kreft D, Stroebel AB, Verbrugh HA (1998) Comparison of eight methods to detect vancomycin resistance in enterococci. J Clin Microbiol 36, 592-594.

14. Griffin PM, Price GR, Schooneveldt JM, , Tilse MH, Urbanski T, Hamilton B, Venter D (2012) Use of matrix-assisted laser desorption ionization-time of flight mass spectrometry to identify vancomycinresistant enterococci and investigate the epidemiology of an outbreak. J Clin Microbiol 50, 2918-2931.

15. Griffin PM, Price GR, Schooneveldt JM, Schlebusch S, Urbanski T, Hamilton B, Venter D (2013) Rapid identification of VRE with MALDI-TOF MS. Pathology 45S1, S57.

16. Özsoy S, İlki A (2017) Detection of vancomycinresistant enterococci (VRE) in stool specimens submitted for Clostridium difficile toxin testing. Med Microbiol 48, 489-492.

17. Lomonte B, Gutiérrez JM, Romero M, Núñez J, Tarkowski A, Hanson LA (1993) An MTT-based method for the in vivo quantification of myotoxic activity of snake venoms and its neutralization by antibodies. J Immunol Methods 161, 231-237.

18. Ghanwate N, Thakare P, Bhise PR, Gawande S (2016) Colorimetric method for rapid detection of oxacillin resistance in Staphylococcus aureus and its comparison with PCR for mec A gene. Sci Rep 6, ID 23013.

19. Dechayont B, Phuaklee P, Chunthorng-Orn J, Poomirat S, Prajuabjinda O, Vilaichone R, Itharat A (2019) Anti-Helicobacter pylori, anti-inflammatory and antioxidant evaluation of crude extracts from Amomum krervanh fruits. ScienceAsia 45, 109-115.

20. Mercier RC, Houlihan HH, Rybak MJ (1997) Pharmacodynamic evaluation of a new glycopeptide, LY333328, and in vitro activity against Staphylococcus aureus and Enterococcus faecium. Antimicrob Agents Chemother 41, 1307-1312.

21. Coombs GW, Pearson JC, Daley DA, Le T, Robinson OJ, Gottlieb T, Howden BP, Johnson PDR, et al (2014) Molecular epidemiology of enterococcal bacteremia in Australia. J Clin Microbiol 52, 897-905.

22. Dutka-Malen S, Evers S, Courvalin V (1995) Detection of glycopeptide resistance genotypes and identification to the species level of clinically relevant enterococci by PCR. J Clin Microbiol 33, 24-27.

23. Humphries RM, Ambler J, Mitchell SL, Castanheira M, Dingle T, Hindler JA, Koeth L, Sei K, et al (2018) CLSI methods development and standardization working group best practices for evaluation of 
antimicrobial susceptibility tests. J Clin Microbiol 56, e0193417.

24. Billington EO, Phang SH, Gregson DB, Pitout JD, Ross T, Church DL, Laupland KB, Parkins MD (2014) Incidence, risk factors, and outcomes for Enterococcus spp. blood stream infections: a population-based study. Int J Infect Dis 26, 76-82.

25. Remschmidt C, Schröder C, Behnke M, Gastmeier P, Geffers C, Kramer TS (2018) Continuous increase of vancomycin resistance in enterococci causing nosocomial infections in Germany - 10 years of surveillance. Antimicrob Resist Infect Control 7, ID 54.

26. Yang JX, Li T, Ning YZ, Shao DH, Liu J, Wang SQ, Liang GW (2015) Molecular characterization of resistance, virulence and clonality in vancomycinresistant Enterococcus faecium and Enterococcus faecalis: A hospital-based study in Beijing, China. Infect Genet Evol 33, 253-260.

27. NARST (2016) Antimicrobial resistance rates of Enterococcus spp. by year (NARST-55 hospitals). National
Antimicrobial Resistance Surveillance Center, Thailand. [Available at: http://narst.dmsc.moph.go.th/ data/AMR\%202000-2016.pdf.]

28. Mosmann T (1983) Rapid colorimetric assay for cellular growth and survival: application to proliferation and cytotoxicity assays. $J$ Immunol Methods 65, 55-63.

29. Grela E, Kozłowska J, Grabowiecka A (2018) Current methodology of MTT assay in bacteria - A review. Acta Histochem 120, 303-311.

30. Wang H, Cheng H, Wang F, Wei D, Wang X (2010) An improved 3-(4,5-dimethylthiazol-2-yl)-2,5-diphenyl tetrazolium bromide (MTT) reduction assay for evaluating the viability of Escherichia coli cells. $J$ Microbiol Meth 82, 330-333.

31. Hundie GB, Woldemeskel D, Gessesse A (2016) Evaluation of direct colorimetric MTT assay for rapid detection of rifampicin and isoniazid resistance in Mycobacterium tuberculosis. PLoS One 11, e0169188. 